\title{
PREVALÊNCIA DE ENTEROPARASITOSES EM FEZES COLHIDAS DURANTE NECRÓPSIAS
}

\author{
João Antônio de Almeida, Maria Betânia Mahler Araújo, \\ Maria Laura Pinto Rodrigues, Marlene Antônia dos Reis \\ e Vicente de Paula Antunes Teixeira.
}

\begin{abstract}
Com o objetivo de verificarmos a prevalência de enteroparasitoses, colhemos o conteúdo do tubo digestivo, em três porções distintas, durante a realização de 135 necrópsias completas. As fezes foram conservadas em MIF (mistura de formol, iodo e merthiolate), e analisadas pelo exame protoparasitológico direto. Encontramos enteroparasitas em 40 casos (29,6\%), sendo que em 11 deles (27,5\%) havia poliparasitismo e em 29 casos (72,5\%) monoparasitismo. As enteroparasitoses mais freqüentes foram o $\mathrm{S}$. stercoralis $(31,9 \%)$, E. histolytica (23,4\%), Ancilostomídeos $(19,1 \%)$ e A. lumbricoides (17\%). Os resultados estāo de acordo com levantamentos epidemiológicos na região. Além disso, mostram que o exame protoparasitológico de fezes colhidas concomitantemente ao exame necroscópico fornece mais um subsidio ao diagnóstico anátomopatológico.
\end{abstract}

Palavras-chaves: Exame protoparasitológico de fezes. Necrópsias. Enteroparasitoses. Coprologia.

As enteroparasitoses ocupam importante papel na morbidade da população nos paises em desenvolvimento. Participam efetivamente de uma gama variável de manifestações próprias do aparelho digestivo e de outros sistemas, às vezes contribuindo direta ou indiretamente para a causa de morte 1479141617 .

Inúmeros levantamentos epidemiológicos feitos no Brasil2 5813 confirmam sua importância, alertando a prática médica a este respeito. Curiosamente, são poucos os levantamentos em que foram utilizados protocolos de necrópsias, talvez porque nestas geralmente explora-se muito pouco as enteroparasitoses. Restringem-se aos registros de infecções maciças ${ }^{11} 12$, de localizações insólitas ${ }^{15}$ ou de situações quando o homem se comporta como hospedeiro intermediário $^{3} 10$.

No intuito de contribuir com estes levantamentos nos propusemos a avaliar em material de necrópsia a ocorrência de enteroparasitoses. Entendemos que as fezes colhidas em necrópsias poderiam representar uma amostra mais fiel da incidência real destes agentes. Permitiriam ainda complementar o exame microscópico de fezes com o macroscópico de todo o conteúdo do tubo digestivo.

Trabalho realizado na Disciplina de Patologia Geral da Faculdade de Medicina do Triângulo Mineiro, Uberaba, MG.

Endereço para correspondência: Dr. Vicente de Paula Antunes Teixeira. Disciplina de Patologia Geral/FMTM. Pça. Manoel Terra s/n 38015 Uberaba, MG.

Recebido para publicação em 17/09/90.

\section{MATERIAL E MÉTODOS}

Foram estudadas 135 necrópsias completas realizadas no Hospital Escola da Faculdade de Medicina do Triângulo Mineiro (FMTM), em Uberaba, Minas Gerais, de individuos com idade variando de seis meses a 84 anos (45,1 $\pm 19,8$ anos), sendo 87 do sexo masculino e 48 do sexo feminino. Após exame macroscópico minucioso do tubo digestivo, colhemos amostras do conteúdo intestinal de três porções distintas: duodeno, jejuno-íleo e colon transverso. Este material foi conservado em MIF (mistura de formol, iodo e merthiolate) em partes iguais e, pelo menos três lâminas de cada amostra de fezes de cada localização foram examinadas pelo método direto. Além disso, colhemos, processamos e examinamos, rotineiramente, os fragmentos teciduais de todos os órgãos colhidos à necrópsia.

\section{RESULTADOS}

A Tabela 1 mostra a freqüência e a proporção de exames protoparasitológicos positivos de acordo com o sexo do indivíduo. A distribuição das enteroparasitoses nas diferentes faixas etárias pode ser observada na Tabela 2. Em nove necrópsias encontramos vermes adultos no exame macroscópico (em cinco casos Ascaris lumbricoides e em quatro Ancilostomídeos). Embora em três destes casos, o exame microscópico tenha sido negativo, o achado de vermes adultos nas necrópsias permitiu o diagnóstico exclusivamente 
Almeida JA, Araújo MBM, Rodrigues MLP, Reis MA, Teixeira VPA. Prevalência de enteroparasitoses em fezes colhidas durante necrópsias. Revista da Sociedade Brasileira de Medicina Tropical 24: 27-29, jan-mar, 1991

macroscópico de enteroparasitose (em dois casos $A$. lumbricoides e em um caso Ancilostomídeos).

Tabela 1 - Ocorrência de enteroparasitoses em necrópsias, distribuidas de acordo com o sexo.

\begin{tabular}{lccc}
\hline & No de Casos & Positivos & Negativos \\
\hline Masculino & 87 & $30(34,5 \%)$ & $57(65,5 \%)$ \\
Feminino & 48 & $10(20,8 \%)$ & $38(79,2 \%)$ \\
\hline Total & 135 & $40(29,6 \%)$ & $95(70,4 \%)$ \\
\hline
\end{tabular}

$\left(X^{2}=2,7 ; p>0,05\right)$
Embora a freqüência de positividade tenha sido maior entre os individuos do sexo masculino $(34,5 \%$ contra $20,8 \%$ no sexo feminino), verificamos que esta diferença não foi estatisticamente significativa e está de acordo com o observado por outros autores em pesquisa de campo 2 . A maioria das necrópsias que são feitas neste Serviço é de individuos falecidos após a 4 a década; este fato justificaria, pelo menos em parte, a maior freqüência de exames positivos nestas faixas etárias.

Verificamos que apesar da colheita de fezes ter sido feita nos locais descritos como habitat preferencial de alguns enteroparasitas, não houve um aumento importante na freqüência de positividade de

Tabela 2 - Distribuição de enteroparasitoses observadas em necrópsias de acordo com a faixa etária.

\begin{tabular}{lcccccccc}
\hline \multicolumn{1}{c}{ Parasitas } & \multicolumn{7}{c}{ Faixa etária (em anos) } \\
& Até 10 & $10-20$ & $20-30$ & $30-40$ & $40-50$ & $50-60$ & 60 ou mais & Total* \\
\hline Giardia lamblia & 0 & 0 & 0 & 1 & 0 & 0 & 0 & $1(2,1 \%)$ \\
Entamoeba histolytica & 1 & 1 & 0 & 2 & 4 & 1 & 2 & $11(23,4 \%)$ \\
Ascaris lumbricoides & 1 & 2 & 1 & 2 & 1 & 1 & 0 & $8(17,0 \%)$ \\
Strongyloides stercoralis & 0 & 0 & 2 & 2 & 4 & 1 & 5 & $15(31,9 \%)$ \\
Ancilostomídeos & 0 & 1 & 1 & 2 & 1 & 2 & 2 & $9(19,1 \%)$ \\
Trichocephalus trichiurus & 1 & 2 & 0 & 0 & 0 & 0 & 0 & $3(6,4 \%)$ \\
Negativos & 4 & 2 & 13 & 15 & 15 & 17 & 29 & 95 \\
\hline
\end{tabular}

* O número de casos com parasitas é superior ao observado na Tabela 1 porque em 11 casos $(27,5 \%)$ foi encontrado poliparasitismo e em 29 casos $(72,5 \%)$ monoparasitismo.

\section{DISCUSSÃO}

Até onde pudemos pesquisar na literatura, provavelmente esta seja a primeira tentativa de se utilizar o conteúdo do tubo digestivo, colhido concomitantemente ao ato da necrópsia, para a realização de exame protoparasitológico. Todavia, em um novo campo de estudos, os paleoparasitologistas já fizeram revelações importantes de ovos de helmintos no conteúdo intestinal de múmias egípcias datadas de 1250 a.C. e múmias brasileiras de mais de 300 anos $^{6}$. Nestes relatos verifica-se que este é um bom método para o estudo de enteroparasitoses, mesmo decorrido muito tempo após o obito.

A prevalência geral de enteroparasitoses que encontramos $(29,6 \%)$ é menor do que aquelas descritas em outras pesquisas, regionais ou não, que variam de $40,0 \%$ a $89,7 \%$ entre crianças e adolescentes $^{2} 813 \mathrm{e} \mathrm{em}$ torno de $65,0 \%$ na população em geral $^{5}$. Entretanto, em população geral atendida no Ambulatório da FMTM Franciscon (JU Franciscon: comunicação pessoal, 1989), encontrou uma freqüência de positividade de $27,0 \%$ dos exames, porcentagem bastante próxima à descrita neste trabalho. nossos exames quando comparados aos observados nesta cidade por Franciscon (JU Franciscon: comunicação pessoal, 1989), usando métodos de coleta e de exame convencionais. Talvez, a ocorrência de alterações do conteúdo intestinal no intervalo compreendido entre o óbito e a realização da necrópsia (colheita do material), poderiam influenciar a integridade (preservação) de alguns enteroparasitas. Por outro lado, como os individuos necropsiados estiveram internados por tempo e motivos nosológicos variáveis em hospital, não podemos afastar a possililide de que eventualmente tenham recebido algum tratamento ou ingerido drogas (específicas ou não) que de alguma maneira também alteraram o aspecto diagnóstico, macro ou microscópico, dos agentes infectantes mais comuns. Devemos destacar ainda a importância do exame cuidadoso do conteúdo intestinal na necrópsia, pois em três casos somente o exame macroscópico foi positivo.

Os dados apresentados neste trabalho, se por um lado confirmam a prevalência relativamente alta destas enteroparasitoses em nosso meio, por outro também servem para demonstrar que a colheita de fezes feita por ocasião da necrópsia é um importante 
Almeida JA, Araújo MBM, Rodrigues MLP, Reis MA, Teixeira VPA. Prevalência de enteroparasitoses em fezes colhidas durante necrópsias. Revista da Sociedade Brasileira de Medicina Tropical 24: 27-29, jan-mar, 1991

elemento auxiliar no diagnóstico anátomo-patológico, permitindo complementar os exames necroscópicos.

\section{SUMMARY}

With the intention to verify the frequency of intestinal parasites, we have taken the inside digestive system material from three differents parts of the intestinal tract while we were doing 135 complete necropsies. The stools were kept in MIF (Formol, iodine and merthiolate mixture) being studied in slides by the direct method. We have found intestinal parasites in 40 cases (29.6\%), 11 $(27.5 \%)$ with two or more kinds of parasites and 29 cases (72.5\%) with only one kind of parasite. The most frequent parasites were: S. stercoralis $(31,9 \%)$, E. hystolytica (23.4\%), hook worm (19.1\%) and A. lumbricoides ( $17 \%)$. The results are in according with epidemiological research made in this area. Thus, they prove that the parasitological exam made in feces taken during the necroscopy provides further evidence to enrich the anatomo-pathological diagnosis.

Key-words: Feces parasitological exam. Necropsies. Enteroparasitism. Coprology.

\section{REFERÊNCIAS BIBLIOGRÁFICAS}

1. Bloch M. El eslabon inmunologico perdido, deficiência inmunologica hereditaria en infecciones y enfermedades autoinmunes. Revista de Instituto de Investigaciones Médicas 12: 207-230, 1983.

2. Carneiro JR, Calil F. Incidência de parasitas intestinais em um estudo de amostras aleatórias em crianças e adolescentes no distrito de Senador Canedo - GO. Revista de Patologia Tropical 8: 129-133, 1979.

3. Chapadeiro E, Lopes ER, Mesquita PM. Primary hydatid cysts of the heart. The Journal of Tropical Medicine and Hygiene 68: 9-11, 1965.

4. Cook GA, Rodrigues H, SILVA H, Rodriguez-Iturbe B, Rodriguez HB. Adult respiratory distress secondary to strongyloidiasis. Chest 92: 1115-1116, 1987.

5. Evangelista A, Komma MD, Santos MAQ. Prevalência de parasitos intestinais em Goiânia. Revista de Patologia Tropical 1: 51-61, 1972.
6. Ferreira LF, Araújo AJG, Confalonieri UE. Os parasitos do homem antigo. Ciência Hoje 1; 63-67, 1982.

7. Fraga Filho C, Sobral DT, Arantes MR. Distúrbios da absorçāo intestinal na estrongiloidose. Revista da Sociedade Brasileira de Medicina Tropical 8: 209-216, 1974.

8. Franciscon JU, Prata AR Resultados preliminares sobre inquérito coprológico escolar em Uberaba. In: Resumos do XXIV Congresso da Sociedade Brasileira de Medicina Tropical, Manaus p. 99-100, 1988.

9. Genta RM, Miles P, Fields K. Opportunistic Strongyloides stercoralis infection in lymphoma patients. Report of a case and review of the literature. Cancer 63: 1407$1411,1989$.

10. Gobbi H, Adad S, Neves RR, Almeida HO. Ocorrência de cisticercose (Cysticercus cellulosae) em pacientes necropsiados em Uberaba, MG. Revista de Patologia Tropical 9: 51-59, 1980.

11. Gomes MC. Fatores determinantes de estrongiloidose grave. Jornal Brasileiro de Medicina 30: 47-50, 1976.

12. Gomes MC. Mecanismos patológicos relacionados a auto-endo-infecção na estrongiloidose humana fatal. Revista de Patologia Tropical 9: 165-261, 1980.

13 Moretti IG. Chieffi PP, Nakagawa E, Gomes AC, Foizer ACM. Contribuição ao estudo da história natural de enteroparasitoses em uma comunidade fechada. I. Prevalência de enteroparasitoses em uma comunidade fechada. Revista da Sociedade Brasileira de Medicina Tropical 8: 41-44, 1974.

14. Nery GC, Carvalho VSS, Barbosa LCA. A mortalidade na cidade de Salvador. Revista Baiana de Saúde Pública 10: 7-32, 1983.

15. Prospero JD. Ascaridiose hepática: anatomia patológica, classificação e patogenia. Arquivos dos Hospitais da Santa Casa de São Paulo 6: 1-16, 1960.

16. Rouquayrol MZ, Alencar JE, Sá LCB. Causas de óbito - no municipio de Fortaleza: nomenclatura local e causas de erro. Revista de Medicina da Universidade Federal do Ceará 7: 45-54, 1967.

17. Shelhamer JH, Neva FA, Finn DR. Persistent strongyloidiasis in an immunodeficient patient. The American Journal of Tropical Medicine and Hygiene 31: 746-751, 1982. 